\title{
The beneficial effects of cumulus cells and oocyte-cumulus cell gap junctions depends on oocyte maturation and fertilization methods in mice
}

Cheng-Jie Zhou, Sha-Na Wu, Jiang-Peng Shen, Dong-Hui Wang, Xiang-Wei Kong, Angeleem Lu, Yan-Jiao Li, Hong-Xia Zhou, YueFang Zhao, Cheng-Guang Liang

Cumulus cells are a group of closely associated granulosa cells that surround and nourish oocytes. Previous studies have shown that cumulus cells contribute to oocyte maturation and fertilization through gap junction communication. However, it is not known how this gap junction signaling affects in vivo versus in vitro maturation of oocytes, and their subsequent fertilization and embryonic development following insemination. Therefore, in our study, we performed mouse oocyte maturation and insemination using in vivo- or in vitro-matured oocyte-cumulus complexes (OCCS, which retain gap junctions between the cumulus cells and the oocytes), in vitro-matured, denuded oocytes co-cultured with cumulus cells (DCs, which lack gap junctions between the cumulus cells and the oocytes), and in vitro-matured, denuded oocytes without cumulus cells (DOs). Using these models, we were able to analyze the effects of gap junction signaling on oocyte maturation, fertilization, and early embryo development. We found that gap junctions were necessary for both in vivo and in vitro oocyte maturation. In addition, for oocytes matured in vivo, the presence of cumulus cells during insemination improved fertilization and blastocyst formation, and this improvement was strengthened by gap junctions. Moreover, for oocytes matured in vitro, the presence of cumulus cells during insemination improved fertilization, but not blastocyst formation, and this improvement was independent of gap junctions. Our results demonstrate, for the first time, that the beneficial effect of gap junction signaling from cumulus cells depends on oocyte maturation and fertilization methods. 
1 Title: The beneficial effects of cumulus cells and oocyte-cumulus cell gap junctions depends on 2 oocyte maturation and fertilization methods in mice

3

4 Authors: Cheng-Jie Zhou ${ }^{1}$, Sha-Na Wu ${ }^{1}$, Jiang-Peng Shen ${ }^{1}$, Dong-Hui Wang ${ }^{1}$, Xiang-Wei 5 Kong ${ }^{1}$, Angeleem Lu ${ }^{1}$, Yan-Jiao Li ${ }^{1}$, Hong-Xia Zhou ${ }^{1}$, Yue-Fang Zhao ${ }^{1}$, Cheng-Guang Liang ${ }^{1 *}$ 6

$7{ }^{1}$ The Key Laboratory of National Education Ministry for Mammalian Reproductive Biology and 8 Biotechnology, College of Life Science, Inner Mongolia University, Hohhot, Inner Mongolia, 9 People's Republic of China

11 "Correspondence and reprint requests: Cheng-Guang Liang

12 The Research Center for Laboratory Animal Science, Inner Mongolia University, No. 24 Zhao

13 Jun Road, Hohhot, Inner Mongolia 010070, People's Republic of China

14 Tel: 86-471-3679862

15 Fax: 86-471-3679862

16 Email: liangchengguang@gmail.com

17 
18

19

20

21

22

\section{Abstract}

Cumulus cells are a group of closely associated granulosa cells that surround and nourish oocytes. Previous studies have shown that cumulus cells contribute to oocyte maturation and fertilization through gap junction communication. However, it is not known how this gap junction signaling affects in vivo versus in vitro maturation of oocytes, and their subsequent fertilization and embryonic development following insemination. Therefore, in our study, we performed mouse oocyte maturation and insemination using in vivo- or in vitro-matured oocytecumulus complexes (OCCs, which retain gap junctions between the cumulus cells and the oocytes), in vitro-matured, denuded oocytes co-cultured with cumulus cells (DCs, which lack gap junctions between the cumulus cells and the oocytes), and in vitro-matured, denuded oocytes without cumulus cells (DOs). Using these models, we were able to analyze the effects of gap junction signaling on oocyte maturation, fertilization, and early embryo development. We found that gap junctions were necessary for both in vivo and in vitro oocyte maturation. In addition, for oocytes matured in vivo, the presence of cumulus cells during insemination improved fertilization and blastocyst formation, and this improvement was strengthened by gap junctions. Moreover, for oocytes matured in vitro, the presence of cumulus cells during insemination improved fertilization, but not blastocyst formation, and this improvement was independent of gap junctions. Our results demonstrate, for the first time, that the beneficial effect of gap junction signaling from cumulus cells depends on oocyte maturation and fertilization methods. 


\section{Introduction}

Cumulus cells are defined as a group of closely associated granulosa cells that surround the oocyte and participate in the processes of oocyte maturation and fertilization. Cumulus cell function is dependent on gap junctions that form between cumulus cells and oocytes. Gap junctions are formed by aggregates of intercellular membrane channel proteins called connexins, which are members of a homologous family of more than 20 proteins, and which allow for the exchange of small molecules between adjacent cells (Kidder \& Mhawi 2002).

Fully grown oocytes become competent to undergo two aspects of maturation: cytoplasmic and nuclear. Both of these processes are essential for the formation of an oocyte with the capacity to undergo fertilization and development to live offspring. Nuclear maturation encompasses the processes of reversing meiotic arrest at prophase I and driving the progression of meiosis to metaphase II. Cytoplasmic maturation refers to the processes that prepare the egg for activation and preimplantation development (Eppig 1996).

Gap junctions between cumulus cells and oocytes are thought to be essential for oocyte maturation and fertilization. During oocyte nuclear maturation, gap junctions are the main connection between cumulus cells and oocytes (Feng et al. 2013; Furger et al. 1996; Santiquet et al. 2012), and they allow a rapid transfer of small metabolites and regulatory molecules from the cumulus cells into the oocyte (Van Soom et al. 2002). During in vitro maturation (IVM), the gap junction protein $\mathrm{Cx} 43$ plays a functional role in gap junction breakdown in the oocyte-cumulus complexes (OCCs) (Sasseville et al. 2009). During fertilization, the cumulus cells attract (Eisenbach 1999), trap (Bedford \& Kim 1993), and select spermatozoa (Carrell et al. 1993), and prevent the premature hardening of the zona pellucida (ZP) (Tanghe et al. 2002), all of which are necessary for successful fertilization. Furthermore, gap junctional communication between oocytes and cumulus cells has been shown to be an essential factor in supporting fertilization (Tanghe et al. 2003). To some extent, early embryo development depends on the successful coordination of processes that occur during oocyte cytoplasmic maturation, including molecular 
63 changes, organelle reorganization, and cytoskeletal changes (Damiani et al. 1996; Reyes \& Ross 64 2015; Salamone et al. 2001). Numerous studies have shown that the presence of cumulus cells 65 can improve cytoplasmic maturation (Ikeda \& Yamada 2014; Tanghe et al. 2002). However, it is 66 still not clear if cumulus cells and their gap junctions with oocytes are necessary for the 67 successful in vivo and in vitro maturation of oocytes. Furthermore, there is currently a lack of 68 understanding of the contribution of cumulus cells and gap junctions to fertilization and early 69 embryo development downstream of oocyte maturation.

Thus, in this study, we used four types of oocytes for our maturation and insemination procedures, including in vivo- or in vitro-matured oocyte-cumulus complexes (OCCs, which possess cumulus cells and intact gap junctions), in vitro-matured, denuded oocytes co-cultured

73 with cumulus cells (DCs, which have cumulus cells, but lack gap junctions), and in vitro74 matured, denuded oocytes without cumulus cells (DOs). Using these culture models, we 75 investigated oocyte maturation, fertilization, and early embryo development to evaluate contributions of cumulus cells and oocyte-cumulus cell gap junctions to each of these processes. 


\section{Materials and methods}

\section{Ethics statement and animal feeding regimens}

All studies adhered to procedures consistent with the National Research Council Guide for the Care and Use of Laboratory Animals and were approved by the Institutional Animal Care and Use Committee at the Inner Mongolia University (Approval number: SYXK 2014-0002). Mice were maintained under the care of the Laboratory Animal Facility at Inner Mongolia University. Mice were kept at a constant temperature of $22 \pm 2{ }^{\circ} \mathrm{C}$ on a 12 hour light/dark cycle and had unrestricted access to food and water.

\section{Oocyte collection}

Adult female (B6D2) F1 mice (4-8 weeks of age) were used for the oocyte collections. All chemicals and media were purchased from Sigma-Aldrich Company (St. Louis, MO, USA) unless stated otherwise. The germinal vesicle $(\mathrm{GV})$ stage oocytes were collected by puncturing the follicles of ovaries at 48 hours after pregnant mare serum gonadotropin (PMSG; SanSheng, Ningbo, China) injection. The cumulus cells were removed by gentle pipetting. For in vivo metaphase II (MII) stage oocyte collection, mice were superovulated by injection of 10 IU PMSG, followed by injection of 10 IU human chorionic gonadotropin (hCG; SanSheng, Ningbo, China) 48 hours later. The cumulus cells were dispersed by $0.3 \mathrm{mg} / \mathrm{mL}$ hyaluronidase in HEPESM2 medium.

\section{Oocyte maturation}

GV oocytes were cultured in MEM Alpha ( $\alpha$-MEM; Gibco, Pleasanton, CA, USA) medium supplemented with 5\% (v/v) fetal calf serum (FCS), $10 \mathrm{ng} / \mathrm{mL}$ epidermal growth factor (EGF), and $0.01 \mathrm{AU} / \mathrm{mL}$ follicle-stimulating hormone $(\mathrm{FSH})$, under a humidified atmosphere of $5 \% \mathrm{CO}_{2}$ at $37^{\circ} \mathrm{C}$ for $14-16$ hours. The number of oocytes with the first polar body (PB1) was counted to determine the percentage of nuclear maturation.

\section{IVF and embryo culture}

Adult male (B6D2) F1 mice (12-14 weeks of age) were used for the sperm collections. The sperm suspension was capacitated for 2 hours in $200 \mu \mathrm{L}$ T6 medium supplemented with 10 
104

105

106

107

108

109

110

111

112

113

114

115

116

117

118

119

120

121

122

123

124

125

126

127

128

129

130

$\mathrm{mg} / \mathrm{mL}$ bovine serum albumin (BSA). MII oocytes were incubated with spermatozoa for 6 hours in $200 \mu \mathrm{L}$ T6 medium supplemented with $20 \mathrm{mg} / \mathrm{mL}$ BSA. The sperm concentration used for fertilization was $1 \times 10^{6} / \mathrm{mL}$. The zygotes were collected and cultured in Chatot-Ziomet-Bavister (CZB) medium containing $3 \mathrm{mg} / \mathrm{ml}$ BSA without glucose under a humidified atmosphere of 5\%

$\mathrm{CO}_{2}$ at $37^{\circ} \mathrm{C}$ for the first 2 days, and then transferred to $\mathrm{CZB}$ medium supplemented with 5.5 $\mathrm{mmol} / \mathrm{L}$ glucose when the embryos reached the 4-cell stage. The percentage of embryos that reached the 2-cell stage was used for fertilization evaluation. The embryos were checked at 48 , 72, and 96 hours after fertilization to calculate the percentage of 4-cell, morula, and blastocyst stages, respectively.

\section{Experimental design}

In our study, oocytes were randomly divided into four groups to mature: (1) OCCs from in vivo maturation, which we abbreviated as M-vivo-OCC; (2) GV oocytes with at least three layers of attached cumulus cells for IVM, which we abbreviated as M-vitro-OCC; (3) GV-stage denuded oocytes co-cultured with dispersed cumulus cells (DC) for IVM, which we abbreviated as M-vitro-DC; (4) GV-stage DOs for IVM alone, which we abbreviated as M-vitro-DO. For subsequent insemination, we co-incubated sperm with OCC (I-OCC), DC (I-DC), or DO (I-DO). For cumulus cell and oocyte co-culture, cumulus cells were collected from GV-stage OCCs by pipetting. The cumulus cells collected from each OCC were supplemented back to the corresponding oocyte. The combinations of different maturation procedures and different insemination procedures were tested to evaluate the roles of cumulus, resulting in a total of ten combinations (M-vivo-OCC + I-OCC, M-vivo-OCC + I-DC, M-vivo-OCC + I-DO, M-vitro$\mathrm{OCC}+\mathrm{I}-\mathrm{OCC}, \mathrm{M}$-vitro-OCC + I-DC, M-vitro-OCC + I-DO, M-vitro-DC + I-DC, M-vitro-DC + I-DO, M-vitro-DO + I-DC, and M-vitro-DO + I-DO), which are shown in Figure 1.

\section{Statistical analysis}

The data are presented as the means \pm standard deviation (SD) from three replicate experiments. Differences were evaluated using the Student's t test. $P<0.05$ was regarded as statistically significant. 
131

132

133

134

135

136

137

138

139

140

141

142

143

144

145

146

147

148

149

150

151

152

153

154

155

156

157

\section{Results}

\section{The role of cumulus cells and their gap junctions in oocyte maturation}

To investigate the contribution of cumulus cells and their gap junctions during oocyte maturation, PB1 extrusion was calculated using the following maturation models, as shown in Figure 1: M-vivo-OCC, M-vitro-OCC, M-vitro-DC, and M-vitro-DO. Our results showed that the highest nuclear maturation percentage was obtained among the OCC groups, but there was no statistical difference between the in vivo and in vitro OCC models. However, if cumulus cells were removed from the oocytes, PB1 extrusion was significantly decreased $(P<0.01)$, and this decrease was not reversed by co-culturing cumulus cells with oocytes $(P<0.01)$ (Fig. 2 and Table S1), suggesting that intact gap junctions between the cumulus cells and oocytes are necessary for efficient oocyte maturation.

\section{The role of cumulus cells and their gap junctions in oocyte fertilization}

Fertilization can be affected by both oocyte maturation and insemination procedures. Thus, to assess the effects of cumulus cells and gap junctions during maturation and insemination, we normalized the fertilization percentage by quantifying the number of 2-cell embryos relative to the number of total GV-stage oocytes or total MII-stage oocytes in each group.

Fertilization based on the number of GV oocytes

Using the same maturation method, we observed significant increases in the percentage of 2-cell-stage embryos when comparing oocytes cultured with, to those cultured without, cumulus cells during insemination $(P<0.01)$ (Fig. 3A and Table S2), suggesting that the presence of cumulus cells improves fertilization. For the oocytes matured in vivo (M-vivo-OCC), detachment of the cumulus cells from the oocytes before insemination, which disrupted the gap junctions between the cells, reduced the fertilization percentage $(P<0.01)$. However, under the same conditions, fertilization was not affected for the OCCs matured in vitro (M-vitro-OCC) (Fig. 3A and Table S2). 
Next, we analyzed the data to investigate the influence of maturation methods on

159

160

161

162

163

164

165

166

167

168

169

170

171

172

173

174

175

176

177

178

179

180

181

182

183

184

fertilization outcomes. Using the same insemination method, successful fertilization was dependent on the maturation method of the oocytes. For example, the oocytes matured in vivo (M-vivo-OCC) showed the highest fertilization percentage, the OCCs matured in vitro (M-vitroOCC) showed a median fertilization percentage, and the oocytes matured in vitro with dispersed cumulus cells (M-vitro-DC) or without cumulus cells (M-vitro-DO) showed the lowest fertilization percentages $(P<0.01)$ ( Fig. 3A and Table S2).

\section{Fertilization based on the number of MII oocytes}

To evaluate the contribution of cumulus cells and gap junctions during insemination, we quantified the percentage of 2-cell-stage embryos among the total MII oocytes. Using the same maturation method, we found that the presence of cumulus cells in the insemination medium improved the fertilization percentages $(P<0.01)$ (Fig. 3B and Table S3). For the M-vivo-OCC oocytes, the removal of cumulus cells from oocytes during insemination (I-DO) reduced the fertilization percentages; however, this reduction could be partially reversed by the addition of cumulus cells into the insemination medium (I-DC) $(P<0.01)$ (Fig. 3B and Table S3). Using the same insemination method, the oocytes matured in vivo had higher fertilization percentages than those matured in vitro $(P<0.01)$, but the presence of cumulus cells during IVM did not affect fertilization $(P>0.05)$ (Fig. 3B, and Table S3).

\section{The role of cumulus cells and their gap junctions in embryo development}

To investigate the contributions of cumulus cells to early embryo development, we calculated the percentages of 4-cell, morula, and blastocyst stages following insemination. Successful embryo development is determined by three important factors: the quality of MII oocytes (i.e., the progression from GV stage to MII stage), the procedure of insemination (i.e., the progression from MII stage to 2-cell stage), and the development starting from the 2-cell 
185

186

187

188

189

190

191

192

193

194

195

196

197

198

199

200

201

202

203

204

205

206

207

208

209

210

211

stage (i.e., the progression after 2-cell stage). To assess the effects of cumulus cells and gap junctions during maturation, insemination, and early embryo development, we quantified each embryonic stage as a percentage of the total number of GV-stage oocytes, MII-stage oocytes, and 2-cell-stage embryos, respectively.

\section{Embryonic development based on the number of GV oocytes}

For the oocytes matured in vivo (M-vivo-OCC), we observed a significant increase in the percentage of embryos in groups cultured with cumulus cells during insemination, compared with those without cumulus cells. More specifically, the M-vivo-OCC oocytes that retained gap junctions with cumulus cells during insemination (I-OCC) had the highest embryo development percentage, those inseminated in the presence of cumulus cells but without gap junctions (I-DC) showed a median embryo development percentage, and those inseminated in the absence of cumulus cells (I-DO) had the lowest embryo development percentage (4-cell stage: $P<0.01$, Fig. 4A and Table S4; morula stage: $P<0.01$, Fig. 4B and Table S4; and blastocyst stage: $P<0.01$, Fig. $4 \mathrm{C}$ and Table S4).

For the oocytes matured in vitro with cumulus cells (M-vitro-OCC and M-vitro-DC), the loss of oocyte-cumulus cell gap junctions (I-DC) or the absence of cumulus cells (I-DO) during insemination did not affect early embryo development (4-cell stage: $P>0.05$, Fig. 4A and Table S4; morula stage: $P>0.05$, Fig. 4B and Table S4; and blastocyst stage: $P>0.05$, Fig. $4 \mathrm{C}$ and Table S4).

Interestingly, for the oocytes matured in vitro without cumulus cells (M-vitro-DO), the absence of cumulus cells during insemination (I-DO) reduced the percentage of 4-cell embryos $(P<0.05)$ (Fig. 4A and Table S4). However, this absence did not affect formation of the morula $(P>0.05)$ (Fig. 4B and Table S4) or blastocyst stages $(P>0.05)$ (Fig. 4C and Table S4).

For all types of oocytes matured in vitro (M-vitro-OCC, M-vitro-DC, and M-vitro-DO), the presence of cumulus cells during IVM or insemination did not alter blastocyst formation $(P>0.05)$ (Fig. 4C and Table S4). 
212

213

214

215

216

217

218

219

220

221

222

223

224

225

226

227

228

229

230

231

232

233

234

235

236

237

238

Notably, the oocytes matured in vivo (M-vivo-OCC), regardless of the presence or absence of cumulus cells during insemination (I-OCC, I-DC, and I-DO), had higher embryo development percentages than those matured in vitro (M-vitro-OCC, M-vitro-DC, and M-vitro-DO) (4-cell stage: $P<0.01$, Fig. $4 \mathrm{~A}$ and Table S4; morula stage: $P<0.01$, Fig. $4 \mathrm{~B}$ and Table S4; and blastocyst stage: $P<0.01$, Fig. $4 \mathrm{C}$ and Table S4).

\section{Embryonic development based on the number of MII oocytes}

To evaluate the contribution of cumulus cells and gap junctions during insemination, we quantified the percentage of embryos from each stage relative to the total number of MII oocytes. For the in vivo-matured oocytes (M-vivo-OCC), we observed a greater percentage of embryo development when cumulus cells were present during insemination. More specifically, if cumulus cells retained gap junctions with oocytes (I-OCC), the highest embryo development percentage was obtained. If cumulus cells were separated from oocytes, but were still present in the insemination medium (I-DC), a median embryo development percentage was obtained. If cumulus cells were removed from the insemination medium (I-DO), the percentage of embryo development was much lower (4-cell stage: $P<0.01$, Fig. 4D and Table S5; morula stage: $P<0.01$, Fig. 4E and Table S5; and blastocyst stage: $P<0.01$, Fig. 4F and Table S5).

For the oocytes matured in vitro with cumulus cells (M-vitro-OCC and M-vitro-DC), the absence of cumulus cells during insemination (I-DO) did not affect early embryo development (4-cell stage: $P>0.05$, Fig. 4D and Table S5; morula stage: $P>0.05$, Fig. 4E and Table S5; and blastocyst stage: $P>0.05$, Fig. $4 \mathrm{~F}$ and Table S5).

For the oocytes matured in vitro without cumulus cells (M-vitro-DO), the absence of cumulus cells during insemination (I-DO) reduced the percentage of 4-cell embryos $(P<0.05)$ (Fig. 4D and Table S5), but did not affect the percentages of morula or blastocyst embryos (morula stage: $P>0.05$, Fig. 4E and Table S5; blastocyst stage: $P>0.05$, Fig. 4F and Table S5).

For the oocytes matured in vitro (M-vitro-OCC, M-vitro-DC, and M-vitro-DO), the absence of cumulus cells during IVM and insemination (I-DO) did not affect blastocyst formation 
239

240

241

242

243

244

245

246

247

248

249

250

251

252

253

254

255

256

257

258

259

260

261

262

263

264

265

$(P>0.05)$ (Fig. 4F and Table S5).

The oocytes matured in vivo (M-vivo-OCC), regardless of the presence or absence of cumulus cells during insemination (I-OCC, I-DC, and I-DO), had a greater percentage of embryo development than those matured in vitro (M-vitro-OCC, M-vitro-DC and M-vitro-DO) (4-cell stage: $P<0.01$, Fig. $4 \mathrm{D}$ and Table S5; morula stage: $P<0.01$, Fig. $4 \mathrm{E}$ and Table S5; and blastocyst stage: $P<0.01$, Fig. $4 \mathrm{~F}$ and Table S5).

\section{Embryonic development based on number of 2-cell embryos}

To evaluate the contribution of cumulus cells and gap junctions after insemination, we quantified the percentage of embryos from each stage relative to the total number of 2-cell embryos. For the oocytes matured in vivo (M-vivo-OCC), a high percentage of embryo development was dependent on the presence of cumulus cells and their gap junctions with oocytes during insemination. For the M-vivo-OCC oocytes that retained gap junctions with cumulus cells during insemination (I-OCC), we observed the highest embryo development percentage. If cumulus cells were separated from oocytes, but were still present in the insemination medium (I-DC), a median embryo development percentage was obtained. If cumulus cells were removed from the oocytes during insemination (I-DO), the lowest embryo development percentage was obtained (4-cell stage: $P<0.01$, Fig. 4G and Table S6; morula stage: $P<0.01$, Fig. 4H and Table S6; and blastocyst stage: $P<0.01$, Fig. 4I and Table S6).

For the OCCs matured in vitro (M-vitro-OCC), the absence of cumulus cells during insemination (I-DO) did not affect embryo development (4-cell stage: $P>0.05$, Fig. 4G and Table S6; morula stage: $P>0.05$, Fig. $4 \mathrm{H}$ and Table S6; and blastocyst stage: $P>0.05$, Fig. $4 \mathrm{I}$ and Table S6). Similar results were obtained with DOs matured in vitro (M-vitro-DO) (4-cell stage: $P>0.05$, Fig. 4G and Table S6; morula: $P>0.05$, Fig. $4 \mathrm{H}$ and Table S6; and blastocyst: $P>0.05$, Fig. 4I and Table S6).

However, for the oocytes matured in vitro with cumulus cells (M-vitro-DC), the absence of cumulus cells during insemination increased the percentage of 4-cell-stage and blastocyst, but 
266 not morula embryos (4-cell stage: $P<0.05$, Fig. $4 \mathrm{G}$ and Table S6; and morula stage: Fig. $4 \mathrm{H}$ and 267 Table S6; and blastocyst stage: $P<0.05$, Fig. 4I and Table S6).

268 Compared to the oocytes matured in vitro (M-vitro-OCC, M-vitro-DC, and M-vitro-DO), 269 the presence of cumulus cells during insemination (I-OCC and I-DC) increased the percentage of 270 embryo development from in vivo-matured oocytes (M-vivo-OCC) (4-cell stage: $P<0.01$, Fig. 4G 271 and Table S6; morula stage: $P<0.01$, Fig. 4H and Table S6; and blastocyst stage: $P<0.01$, Fig. 4I 272 and Table S6). 
273

274

275

276

277

278

279

280

281

282

283

284

285

286

287

288

289

290

291

292

293

294

295

296

297

298

299

\section{Discussion}

\section{Cumulus cells and oocyte-cumulus cell gap junctions contribute to oocyte maturation}

In this study, the contribution of cumulus cells and oocyte-cumulus cell gap junctions to oocyte maturation was analyzed under different culture conditions, including in vivo or in vitro maturation, with or without cumulus cells or gap junctions. This comprehensive experimental design allowed us to better understand the functions of cumulus cells and oocyte-cumulus cell gap junctions during maturation. Previous studies have shown that gap junctions between cumulus cells and oocytes are the main channels allowing the exchange of ions and small molecules, such as purines (Downs \& Eppig 1986; Eppig et al. 1985) and cyclic adenosine monophosphate (cAMP) (Kumar \& Gilula 1996; Yoshimura et al. 1992), to inhibit resumption of premature oocyte meiotic progression. Our study demonstrates that these gap junctions are also essential for oocytes to achieve higher nuclear maturation rates. We showed that disruption of these junctions impaired PB1 extrusion, and that this impairment could not be rescued by culturing dispersed cumulus cells with oocytes, which is consistent with previous studies showing that co-culturing with dispersed cumulus cells does not improve the nuclear maturation of oocytes (Downs 2001; Luciano et al. 2005; Tao et al. 2008).

In addition, in our study, we used maturation medium containing EGF and FSH. A previous study in rabbit showed that the presence of growth factors in culture systems facilitates nuclear maturation in OCCs but not in DOs (Lorenzo et al. 1996), which is similar to what we observed. For oocyte growth and development in vitro, the gap junctions between cumulus cells and oocytes must be maintained (Eppig 1979; Hashimoto et al. 1998; Tanghe et al. 2002). Once gap junctions are disrupted by removing the cumulus cells, the passage of necessary signaling molecules is interrupted. Consistent with previous data, we demonstrated that gap junction signaling between oocytes and cumulus cells was critical for oocyte maturation.

\section{Cumulus cells and oocyte-cumulus cell gap junctions contribute to oocyte fertilization}

Although cumulus cell function during oocyte maturation has been widely studied, less is known about cumulus cell contribution during insemination. To the best of our knowledge, this 
300

301

302

303

304

305

306

307

308

309

310

311

312

313

314

315

316

317

318

319

320

321

322

323

324

325

326

study is the first to demonstrate that cumulus cells affected fertilization by influencing in vitro oocyte nuclear maturation. Using the same insemination method, successful fertilization depended on the method of oocyte nuclear maturation, with in vivo-matured oocytes showing the highest fertilization percentages. Using the same IVM method, the presence of cumulus cells during insemination resulted in higher cleavage rates. Therefore, our data suggest that in vivo OCC maturation followed by OCC insemination is the optimal combination for obtaining the highest fertilization percentage.

A new finding in our study was that when in vitro-matured MII oocytes were used for fertilization, the presence of cumulus cells during insemination improved the cleavage percentages, even when the gap junctions were destroyed. It has been proposed that gap junctional communication between the oocyte and corona cells is needed for supporting fertilization (Tanghe et al. 2003). We hypothesize that the improved fertilization percentage obtained when the DOs were inseminated in the presence of cumulus cells, but in the absence of gap junctions, was due to secreted factors from the cumulus cells (Guidobaldi et al. 2008; Sun et al. 2005). This hypothesis is quite plausible, as cumulus cells have been shown to secrete chemotactic factors that guide the spermatozoon to the oocyte (Ito et al. 1991; Sun et al. 2005), which increases the chance of fertilization.

Another new finding of our study was that in vivo-matured oocytes achieved higher cleavage percentages than those from IVM, even in the absence of cumulus cells during insemination. To explain these data, we hypothesize that the in vivo-matured oocytes had undergone more complete cytoplasmic maturation than the in vitro-matured oocytes. Moreover, for the oocytes matured in vivo, removal of cumulus cells during insemination (I-DO) reduced the fertilization percentage, but this reduction was partially reversed by the addition of cumulus cells to the insemination medium. Interestingly, for the in vitro-matured OCCs, this reduction was reversed completely by adding cumulus cells to the insemination medium. These data suggest that the gap junctions of the in vitro-matured OCCs are defective, leading to the reduced fertilization percentages during IVF compared with the in vivo-matured OCCs. 
327 Cumulus cells and oocyte-cumulus cell gap junctions contribute to early embryo 328 development

329 There is an existing debate concerning whether or not cumulus cells contribute to oocyte

330

331

332

333

334

335

336

337

338

339

340

341

342

343

344

345

346

347

348

349 cytoplasmic maturation, a process that is necessary for early embryo development. It has been suggested that co-culturing DOs embedded in cumulus cell clumps can improve cytoplasmic maturation (Feng et al. 2013), and denuding mouse oocytes of cumulus cells impairs in vitro cytoplasmic maturation (Ge et al. 2008). However, our data support the concept that cumulus cells do not contribute to cytoplasmic maturation during IVM. These different outcomes may be due to the employment of different species, different standards for evaluating cytoplasmic maturation, or different maturation conditions used in each study.

Our study showed that oocytes matured in vivo were the most suitable for embryo development, indicating that in vivo-matured oocytes possess more complete cytoplasmic maturation than in vitro-matured oocytes. Previously, pronuclear (PN) transfer between in vivomatured and in vitro-matured oocytes was used to examine the nuclear-ooplasm effects on resultant embryo development. The results showed that as long as the ooplasm was derived from in vivo samples, reconstructed embryos had a higher developmental ability regardless of PN origin (Chang et al. 2005). These data showed that the ooplasm is the decisive factor for embryo development, which is consistent with our hypothesis.

In conclusion, our study systematically evaluated the contribution of cumulus cells and oocyte-cumulus cell gap junctions to oocyte maturation, fertilization, and embryo development, using various oocyte maturation and insemination methods. Our results demonstrate that the beneficial effects of cumulus cells and oocyte-cumulus cell gap junctions depend on oocyte maturation and insemination methods. 


\section{References}

Bedford JM, and Kim HH. 1993. Cumulus oophorus as a sperm sequestering device, in vivo. $J$ Exp Zool 265:321-328.

Carrell DT, Middleton RG, Peterson CM, Jones KP, and Urry RL. 1993. Role of the cumulus in the selection of morphologically normal sperm and induction of the acrosome reaction during human in vitro fertilization. Arch Androl 31:133-137.

Chang HC, Liu H, Zhang J, Grifo J, and Krey LC. 2005. Developmental incompetency of denuded mouse oocytes undergoing maturation in vitro is ooplasmic in nature and is associated with aberrant Oct-4 expression. Hum Reprod 20:1958-1968.

Damiani P, Fissore RA, Cibelli JB, Long CR, Balise JJ, Robl JM, and Duby RT. 1996. Evaluation of developmental competence, nuclear and ooplasmic maturation of calf oocytes. Mol Reprod Dev 45:521-534.

Downs SM. 2001. A gap-junction-mediated signal, rather than an external paracrine factor, predominates during meiotic induction in isolated mouse oocytes. Zygote 9:71-82.

Downs SM, and Eppig JJ. 1986. The role of purines in the maintenance of meiotic arrest in mouse oocytes. Tokai J Exp Clin Med 11:463-469.

Eisenbach M. 1999. Sperm chemotaxis. Rev Reprod 4:56-66.

Eppig JJ. 1979. A comparison between oocyte growth in coculture with granulosa cells and oocytes with granulosa cell-oocyte junctional contact maintained in vitro. J Exp Zool 209:345-353.

Eppig JJ. 1996. Coordination of nuclear and cytoplasmic oocyte maturation in eutherian mammals. Reprod Fertil Dev 8:485-489.

Eppig JJ, Ward-Bailey PF, and Coleman DL. 1985. Hypoxanthine and adenosine in murine ovarian follicular fluid: concentrations and activity in maintaining oocyte meiotic arrest. Biol Reprod 33:1041-1049.

Feng G, Shi D, Yang S, and Wang X. 2013. Co-culture embedded in cumulus clumps promotes maturation of denuded oocytes and reconstructs gap junctions between oocytes and cumulus cells. Zygote 21:231-237.

Furger C, Cronier L, Poirot C, and Pouchelet M. 1996. Human granulosa cells in culture exhibit functional cyclic AMP-regulated gap junctions. Mol Hum Reprod 2:541-548.

Ge L, Sui HS, Lan GC, Liu N, Wang JZ, and Tan JH. 2008. Coculture with cumulus cells improves maturation of mouse oocytes denuded of the cumulus oophorus: observations of nuclear and cytoplasmic events. Fertil Steril 90:2376-2388.

Guidobaldi HA, Teves ME, Unates DR, Anastasia A, and Giojalas LC. 2008. Progesterone from the cumulus cells is the sperm chemoattractant secreted by the rabbit oocyte cumulus complex. PLoS One 3:e3040.

Hashimoto S, Saeki K, Nagao Y, Minami N, Yamada M, and Utsumi K. 1998. Effects of cumulus cell density during in vitro maturation of the developmental competence of bovine oocytes. Theriogenology 49:1451-1463.

Ikeda S, and Yamada M. 2014. Midkine and cytoplasmic maturation of mammalian oocytes in the context of ovarian follicle physiology. Br J Pharmacol 171:827-836. 
391

392

393

394

395

396

397

398

399

400

401

402

403

404

405

406

407

408

409

410

411

412

413

414

415

416

417

418

419

420

421

422

423

424

425

426

427

428

429

430

Ito M, Smith TT, and Yanagimachi R. 1991. Effect of ovulation on sperm transport in the hamster oviduct. J Reprod Fertil 93:157-163.

Kidder GM, and Mhawi AA. 2002. Gap junctions and ovarian folliculogenesis. Reproduction 123:613-620.

Kumar NM, and Gilula NB. 1996. The gap junction communication channel. Cell 84:381-388.

Lorenzo PL, Rebollar PG, Illera MJ, Illera JC, Illera M, and Alvarino JM. 1996. Stimulatory effect of insulin-like growth factor I and epidermal growth factor on the maturation of rabbit oocytes in vitro. $J$ Reprod Fertil 107:109-117.

Luciano AM, Lodde V, Beretta MS, Colleoni S, Lauria A, and Modina S. 2005. Developmental capability of denuded bovine oocyte in a co-culture system with intact cumulus-oocyte complexes: role of cumulus cells, cyclic adenosine 3',5'-monophosphate, and glutathione. Mol Reprod Dev 71:389-397.

Reyes JM, and Ross PJ. 2015. Cytoplasmic polyadenylation in mammalian oocyte maturation. Wiley Interdiscip Rev RNA.

Salamone DF, Damiani P, Fissore RA, Robl JM, and Duby RT. 2001. Biochemical and developmental evidence that ooplasmic maturation of prepubertal bovine oocytes is compromised. Biol Reprod 64:1761-1768.

Santiquet NW, Develle Y, Laroche A, Robert C, and Richard FJ. 2012. Regulation of gapjunctional communication between cumulus cells during in vitro maturation in swine, a gap-FRAP study. Biol Reprod 87:46.

Sasseville M, Gagnon MC, Guillemette C, Sullivan R, Gilchrist RB, and Richard FJ. 2009. Regulation of gap junctions in porcine cumulus-oocyte complexes: contributions of granulosa cell contact, gonadotropins, and lipid rafts. Mol Endocrinol 23:700-710.

Sun F, Bahat A, Gakamsky A, Girsh E, Katz N, Giojalas LC, Tur-Kaspa I, and Eisenbach M. 2005. Human sperm chemotaxis: both the oocyte and its surrounding cumulus cells secrete sperm chemoattractants. Hum Reprod 20:761-767.

Tanghe S, Van Soom A, Mehrzad J, Maes D, Duchateau L, and de Kruif A. 2003. Cumulus contributions during bovine fertilization in vitro. Theriogenology 60:135-149.

Tanghe S, Van Soom A, Nauwynck H, Coryn M, and de Kruif A. 2002. Minireview: Functions of the cumulus oophorus during oocyte maturation, ovulation, and fertilization. Mol Reprod Dev 61:414-424.

Tao Y, Cao C, Zhang M, Fang F, Liu Y, Zhang Y, Ding J, and Zhang X. 2008. Effects of cumulus cells on rabbit oocyte in vitro maturation. J Anim Physiol Anim Nutr (Berl) 92:438-447.

Van Soom A, Tanghe S, De Pauw I, Maes D, and de Kruif A. 2002. Function of the cumulus oophorus before and during mammalian fertilization. Reprod Domest Anim 37:144-151.

Yoshimura Y, Nakamura Y, Ando M, Jinno M, Oda T, Karube M, Koyama N, and Nanno T. 1992. Stimulatory role of cyclic adenosine monophosphate as a mediator of meiotic resumption in rabbit oocytes. Endocrinology 131:351-356. 


\section{1}

Experimental design.

In vivo MII stage oocyte-cumulus complexes (OCC) were collected from oviduct in mice superovulated by PMSG, followed by hCG. GV stage OCCs were collected from ovaries of the mice $48 \mathrm{~h}$ after the administration of PMSG. Cumulus cells were removed by gentle pipetting. Oocytes were divided into four groups for maturation: in vivo OCC, in vitro OCC, in vitro denuded oocyte (DO) + cumulus cells, and in vitro DO. For OCCs matured in vivo or in vitro, oocytes were divided into three groups for insemination: OCC, DO + cumulus, and DO. For in vitro matured DOs with or without cumulus cells, oocytes were divided into two groups for insemination: DO + cumulus and DO. The abbreviations used are listed at far right of each maturation and insemination model. Scale $=50 \mu \mathrm{m}$. 

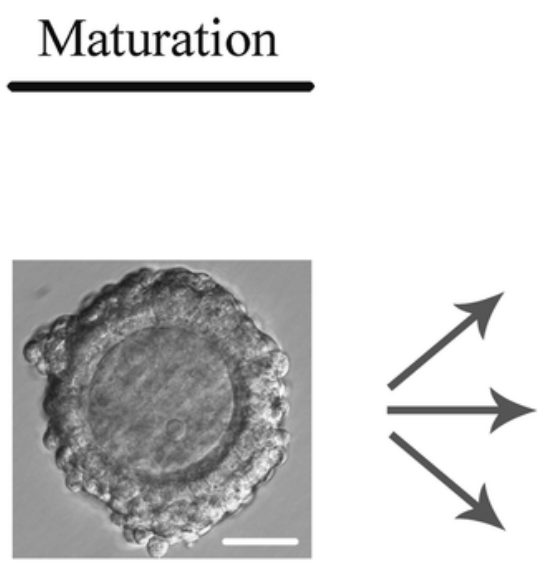

in vivo oocyte-cumulus complex

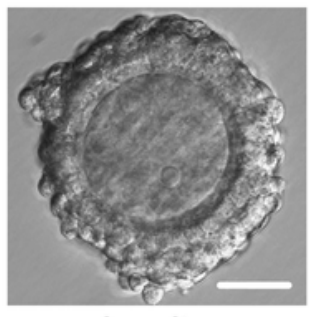

in vitro oocyte-cumulus complex

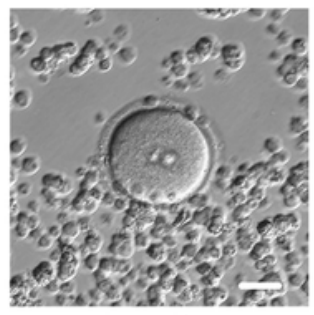

in vitro

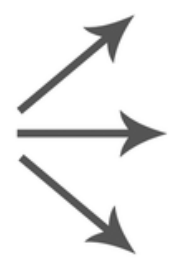

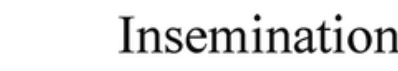
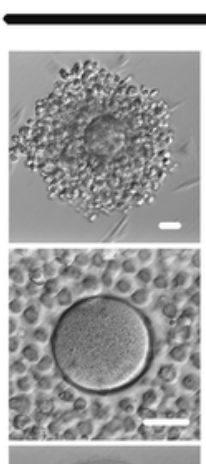

denuded oocyte + cumulus

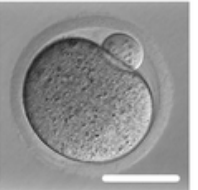

denuded oocyte

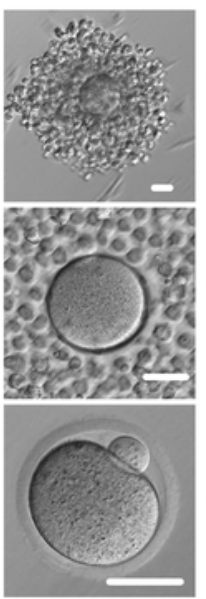

oocyte-cumulus complex

denuded oocyte + cumulus

denuded oocyte M-vitro-OCC + I-DO

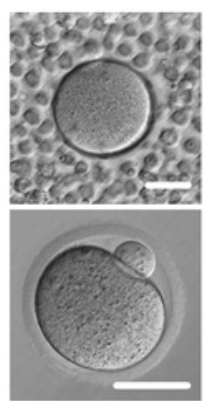

denuded oocyte

+ cumulus

M-vitro-DC + I-DC

denuded oocyte M-vitro-DC + I-DO

Abbreviation

M-vivo-OCC + I-OCC

M-vivo-OCC + I-DC

M-vivo-OCC + I-DO

M-vitro-OCC + I-OCC

M-vitro-OCC + I-DC

denuded oocyte

+ cumulus

M-vitro-DO + I-DC

denuded oocyte M-vitro-DO + I-DO

denuded oocyte + cumulus

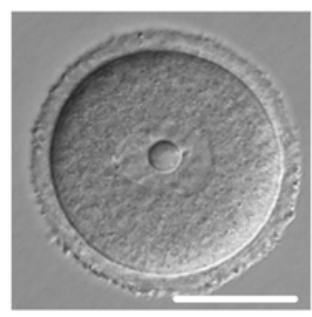

in vitro denuded oocyte
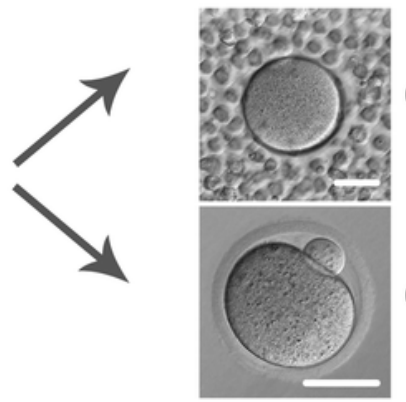
2

Percentof PB1 under different maturation methods .

Oocytes were divided into four groups for maturation: M-vivo-OCC, M-vitro-OCC, M-vitro-DC, and M-vitro-DO. The percentages of the PB1 were calculated. Percentages without a common letter are statistical significant different $(P<0.05)$.

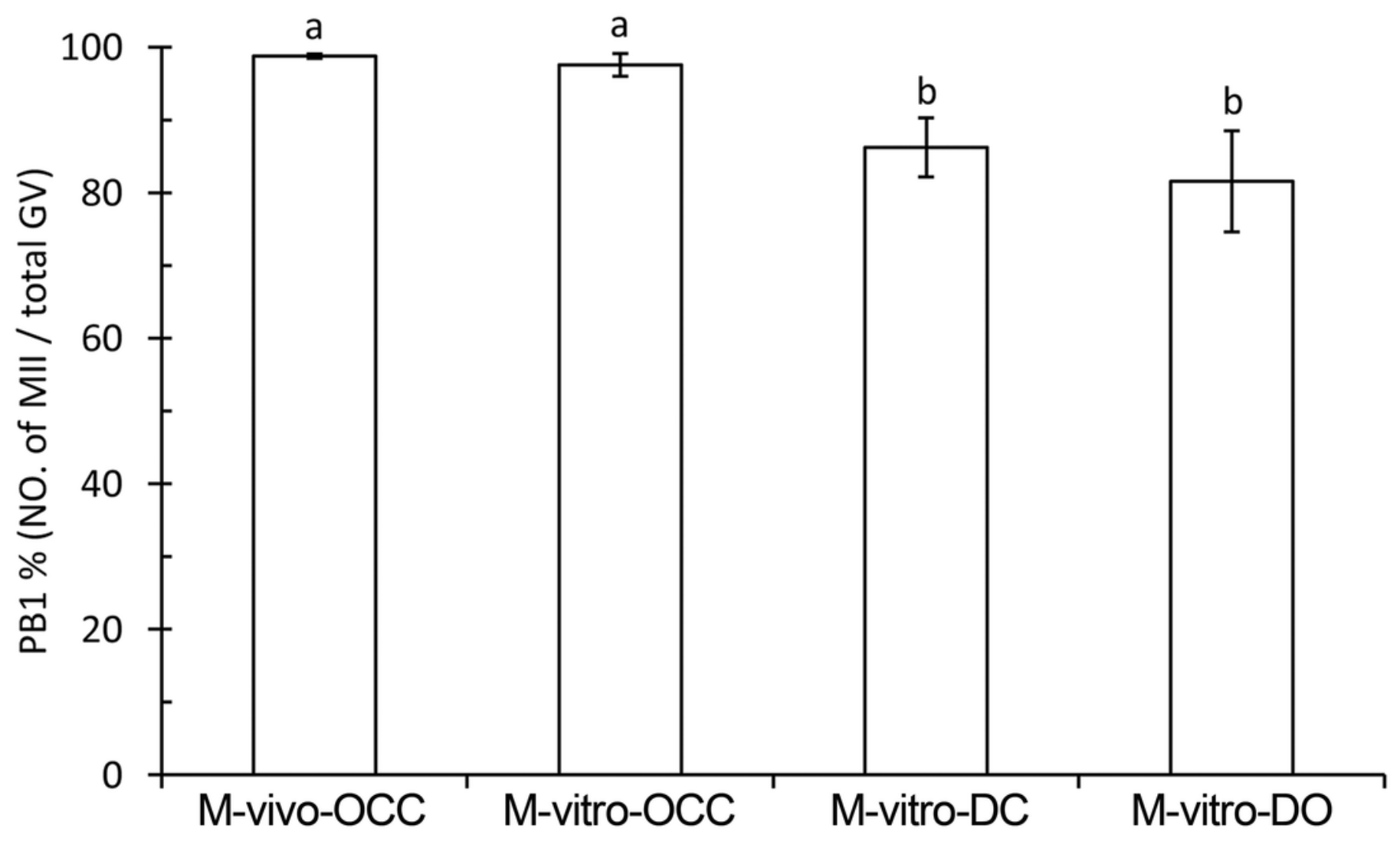


3

Fertilizationof oocytes under different maturation and insemination combinations .

(A) Percent of fertilization based on the number of GV oocytes. (B) Percent of fertilization based on the number of MII oocytes. Percentages without a common letter are statistical significant different $(P<0.05)$. 

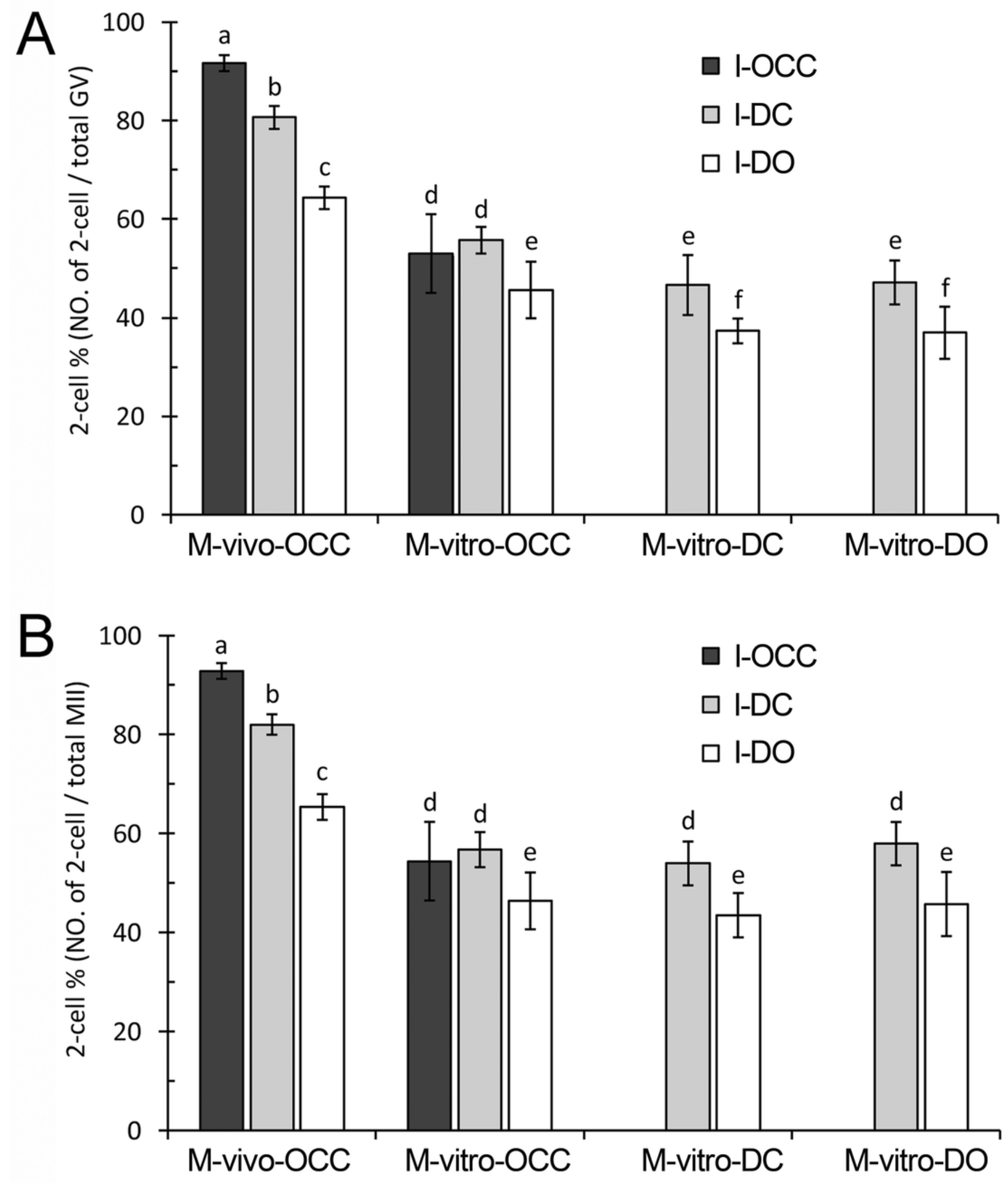
4

Developmentof embryos generated from combinations of different maturation and inseminationmethods.

(A), (B) and (C), Embryonic developm ent based on the number of GV oocytes. (D), (E) and (F), Embryonic development based on the num ber of MII oocytes. (G), (H) and (I), Embryonic development based on number of 2-cell embryos. Percentages without a common letter are statistical significant different $(P<0.05)$.
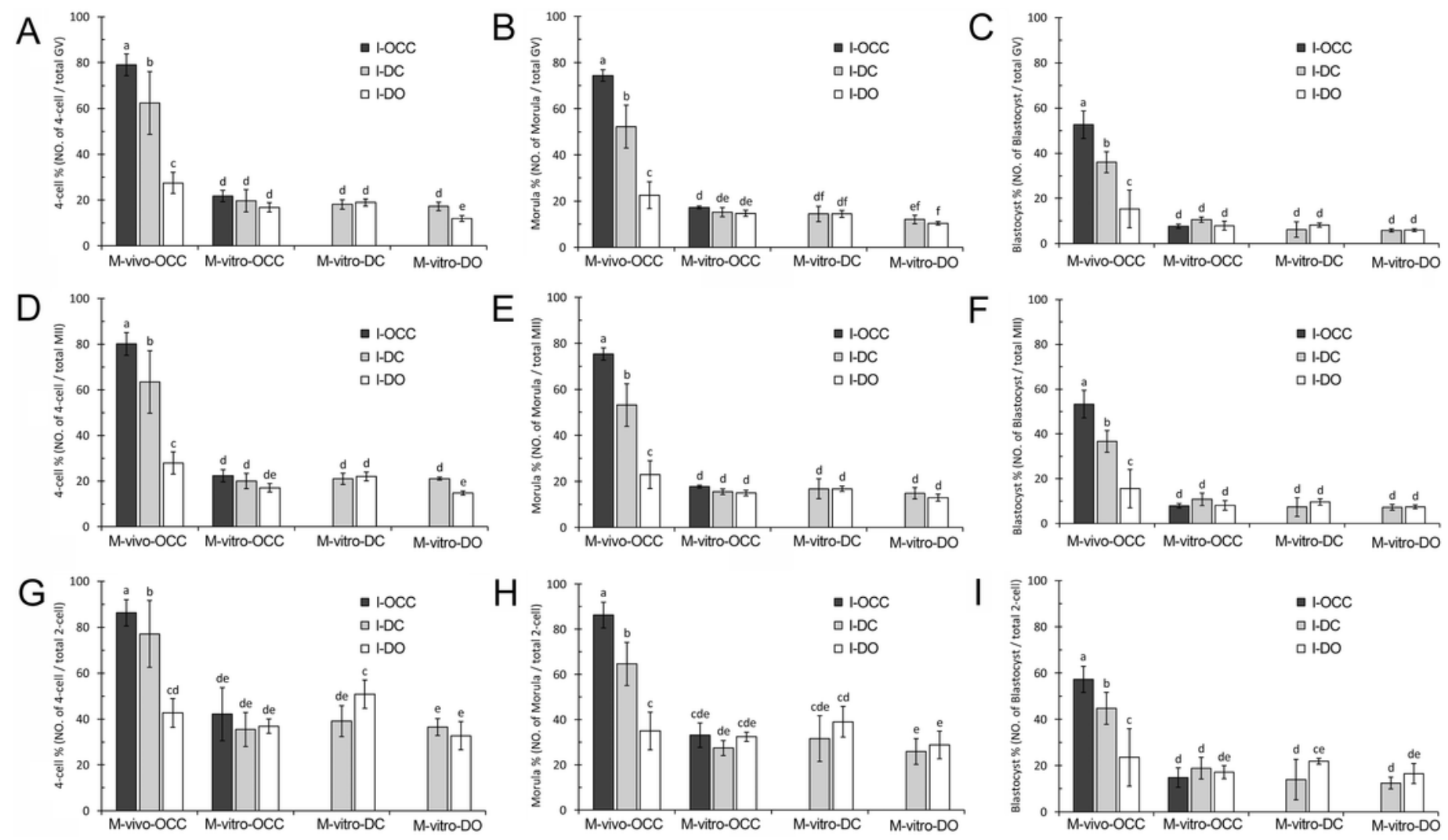